\title{
Post-Surgical Outcomes After Sternal Closure Using Zipfix Band and Steel Wires
}

\author{
Udgeath Dhir $^{1, ~ *, ~ A n a n d ~ K u m a r ' ~}{ }^{1}$, Rakesh Saklani ${ }^{1}$, Vishal Jain ${ }^{2}$ \\ ${ }^{1}$ Department of Cardiothoracic and Vascular Surgery, Fortis Memorial Research Institute, Gurgaon, India \\ ${ }^{2}$ Department of Cardiac Anaesthesia, Fortis Memorial Research Institute, Gurgaon, India

\section{Email address:} \\ dhirudgeath@gmail.com (U. Dhir), dranand_K1@yahoo.co.uk (A. Kumar), saklanirakesh10@gmail.com (R. Saklani), \\ vishal_jain@yahoo.com (V. Jain) \\ ${ }^{*}$ Corresponding author
}

\section{To cite this article:}

Udgeath Dhir, Anand Kumar, Rakesh Saklani, Vishal Jain. Post-Surgical Outcomes After Sternal Closure Using Zipfix Band and Steel Wires. International Journal of Cardiovascular and Thoracic Surgery. Vol. 6, No. 6, 2020, pp. 85-89. doi: 10.11648/j.ijcts.20200606.15

Received: November 30, 2020; Accepted: December 19, 2020; Published: December 25, 2020

\begin{abstract}
Median sternotomy is the most commonly used and the standard way of an incision after cardiothoracic procedures. The use of stainless steel wire has been considered the gold standard in the sternal closure system; however, increasing postoperative complications have caused other options. The aim of this study is to understand the combined effect of the ZipFix bands with steel wires on postoperative outcomes after sternal closure. This is a real-world evidence study of patients treated using the ZipFix band and steel wires. The sternal closure is performed by fixing of manubrium with straight simple wires, followed with ZipFix 3 or 4 spaces. Demographic characters of patients using the ZipFix band and steel wire and their relation to sternal infections, wound dehiscence, and post-surgical outcomes are studied. From gathered evidence, $2 \%$ of patients' cases were complicated with the incidence of sternal infections. Ninety-nine percentage of patients had a stable and healthy wound while $1 \%$ of patients showed sternal wound dehiscence. The mean duration of hospital and intensive care unit stay was $8.53( \pm 4.84)$ days and $3.58( \pm 5.01)$ days respectively. The use of the sternal ZipFix system in combination with stainless steel wire is found to be effective in reducing post-surgical complications.
\end{abstract}

Keywords: Zipfix, Median Sternotomy, Stainless Steel Wires, Sternal Wound Infections

\section{Introduction}

Accelerated rise in cardiac diseases due to an increase in life expectancy has caused a rise in the minimally invasive methods in open-heart surgery. [1] Median sternotomy is the standard way of an incision after cardiothoracic procedures and the preferred technique owing to its low incidence of complications and less painful nature. Many prospective and retrospective outcome studies have identified that poststernotomy complications such as dehiscence, mediastinitis, osteomyelitis, and sternal wound infection (SWI) may be rare (1 to $10 \%$ ) but can result in considerable morbidity and mortality (19 to $29 \%$ ) and may have significant economic implications. [1-5]

Conservatively, stainless steel (SS) wires are used for closure of the sternal system due to their easy to use and inexpensive nature. [6] However, many studies have reported the post- surgical drawbacks of using SS wires such as poor sternal healing, dehiscence, poor rigidity, and SWI. [6-8] These postsurgical complications can intricate the recovery by prolonging hospitalization; they may increase morbidity and mortality. A few innovations in closure techniques are attempted over the period such as reinforced wires, sternum cable, and plating. They are developed to minimize the risk of non-union, provide sternal stability, especially in high-risk patients. [2]

Many devices didn't succeed due to discordancy in practicability and economy. The sternal ZipFix, a cable tie sternal closure system, made of biocompatible fiber polyether-ether-ketone (PEEK) [7] have shown excellent stress and fatigue resistance properties through various biomedical studies. [2] Various studies have reported the use of sternal ZipFix has decreased post-surgical complications such as sternal dehiscence and SWI and provided stability to the sternum. $[2,6-8]$ It is also relatively cost-effective. [8]

Cable-tie closure system, in combination with wire sutures, 
is a simple and reliable method for fastening together the corpus sterni after median sternotomy. Post-surgical outcomes, the major concern in using SS wires can also be managed with the use of a cable tie sternum closure system along with SS wires. As reported by Stelly et al., the use of cable ties may reduce the risk of DSWI compared to wire sutures alone. [8] This study collates the evidence of the effects of the ZipFix system along with SS wire on post-surgical complications such as sternal infections and wound complications.

\section{Materials and Methods}

This was a real-world evidence study with 100 patients undergoing cardiac surgeries at Fortis Memorial Research Institute (FMRI), Gurgaon between May 2018 and December 2018. The patients above 18 years of age and who underwent cardiac surgery were included in the study. The population criteria excluded the patients, who were pregnant, who were under 18 years old, and who had a previous history of cardiac surgery, or have/had any autoimmune disease or connective tissue disorder or sternal infections. Six months of follow-up data were considered for the analysis. The data points such as medical history, pre-operative data, surgery details, and postoperative complications were collected. The ethics committee approval was not required for this study.

\subsection{Surgical Technique}

The sternal closure is performed by fixing of manubrium with straight simple wires, followed with the ZipFix 3 spaces or 4 spaces. The simple principle is fixing the lower intercostal space with simple wire and figure of eight. The figure of eight wire is used to prevent rotational force at false ribs. The false ribs have to be reinforced more to bear the brunt of more torsional forces. The basic principle of sternal closure is to fix the manubrium and lower end for both rotational and longitudinal strain. Simple ' 7 ' wires are dictum of weight for a person weighing an average weight of $70 \mathrm{~kg}$ and an extra one wire is given for every extra $10 \mathrm{~kg}$.

\subsection{Statistical Analysis}

All variables were assessed for normality. For statistical analysis, results were presented as mean (standard deviation (SD)) for quantitative variables and were presented by absolute frequencies and percentages for categorical variables. Categorical variables were compared using Fisher's exact test where numbers were small and were reported as mean, median, and standard deviation. Continuous variables were compared using the nonparametric Mann-Whitney test for the data which had nonnormal distribution. All statistical analysis was performed using IBM SPSS statistics 20 software.

\section{Results}

The data of a total of 100 patients were recorded in this realworld evidence study. They had undergone the sternal closure with SS wires and ZipFix cable wires. The baseline characteristics of the patients are shown in Table 1 . The mean age of the population was 59.23 (SD: 9.50 ) years with $85 \%$ of males. The mean body mass index (BMI) was 27.81 (SD: $5.61 \mathrm{~kg} / \mathrm{m}^{2}$ ). Preoperative variables such as ejection fraction (EF) and logistic euro score were recorded for each patient and the mean is mentioned in table 1. Major comorbidities accompanied to patients were dyslipidemia (80\%), coronary artery disease $(76 \%)$, hypertension $(70 \%)$, diabetes mellitus $(59 \%)$, triple vessel disease (TVD) (44\%), smoking (31\%), and other diseases.

Table 1. Demographic characteristics of patients.

\begin{tabular}{lll}
\hline Baseline characteristic & Mean & Standard deviation \\
\hline Age $($ years $)$ & 59.23 & 9.50 \\
BMI $\left(\mathrm{kg} / \mathrm{m}^{2}\right)$ & 27.81 & 5.61 \\
Logistic Euro Score & 2.84 & 2.78 \\
Ejection Fraction & 43.75 & 9.41 \\
\hline
\end{tabular}

Operation types included CABG (X3 grafts) (31 patients), CABG (X2 grafts) (15 patients), CABG (X1 grafts) (4 patients), combined CABG with other procedures CABG (24 patients), aortic operations ( 3 patients), and other procedures (23 patients).

From the total number of patients, 98 (98\%) patients didn't develop any sternal infections. However, 2 (2\%) patients' cases were complicated with the incidence of sternal infections (superficial or deep) over a follow-up period of 6 months. Dehiscence was too low among the patients i.e 1 (1\%) patient showed sternal wound dehiscence (Table 2).

Table 2. The incidence rate of sternal wound complications.

\begin{tabular}{ll}
\hline Sternal infections (superficial or deep) & Frequency (Percent) \\
\hline No & 98.0 \\
Yes & 2.0 \\
Sternum wound condition & Frequency (Percent) \\
Stable and healthy wound & 99.0 \\
Wound dehiscence & 1.0 \\
\hline
\end{tabular}

These sternal infections and wound conditions were associated with demographic factors such as age and BMI. There was no significant difference in the age of the patients with and without sternal infection $(p=0.20)$. Similarly, no statistically significant difference was found in the age of patients in different wound conditions (healthy and stable wound and wound dehiscence) $(\mathrm{p}=0.14)$ (Table 3$)$.

Table 3. The association of age with sternal wound complications.

\begin{tabular}{llll}
\hline & No & Mean age & 59.39 \\
$\begin{array}{l}\text { Sternal infections } \\
\text { (superficial or }\end{array}$ & & Std. Deviation & 9.47 \\
deep) & Yes & Mean age & 51.50 \\
& & Std. Deviation & 10.61 \\
& & p Value & 0.20 \\
Sternum wound & Stable and & Mean age & 59.38 \\
condition & healthy wound & Std. Deviation & 9.42 \\
& Wound & Mean age & 44.00 \\
& dehiscence & Std. Deviation & \\
& & p Value & 0.14 \\
\hline
\end{tabular}

There was no significant difference in BMI of patients with stable and healthy wounds and patients with wound dehiscence $(p=0.14)$. The presence of sternal infections and their association 
with BMI was not statistically significant $(\mathrm{p}=0.92)$ (Table 4).

Table 4. The association of BMI with sternal wound complications.

\begin{tabular}{llll}
\hline & \multirow{2}{*}{ No } & Mean BMI & 27.76 \\
Sternal infections [superficial & & Std. Deviation & 5.64 \\
or deep] & Yes & Mean BMI & 30.45 \\
& & Std. Deviation & 4.31 \\
& & p Value & 0.335 \\
& & Mean BMI & 27.82 \\
Sternum wound condition & Stable and healthy wound & Std. Deviation & 5.64 \\
& Wound dehiscence & Mean BMI & 27.40 \\
& & Std. Deviation & 0.92 \\
\hline
\end{tabular}

Smoking behavior of patients and its relation to the presence of sternal infection $(\mathrm{p}=0.53)$ and wound condition was not statistically significant $(\mathrm{p}=0.69)$ (Table 5).

Table 5. The association of smoking history with sternal dehiscence.

\begin{tabular}{|c|c|c|c|c|c|}
\hline & & \multicolumn{2}{|l|}{ Smoking } & \multirow{2}{*}{ Total } & \multirow{2}{*}{ p Value } \\
\hline & & No & Yes & & \\
\hline $\begin{array}{l}\text { Sternal infections (superficial or } \\
\text { deep) }\end{array}$ & $\begin{array}{l}\text { No } \\
\text { Yes }\end{array}$ & $\begin{array}{l}68(98.55) \\
1(1.45)\end{array}$ & $\begin{array}{l}30(96.77) \\
1(3.23)\end{array}$ & $\begin{array}{l}98(98) \\
2(2)\end{array}$ & 0.53 \\
\hline Sternum wound condition & $\begin{array}{l}\text { Stable and healthy wound } \\
\text { Wound dehiscence }\end{array}$ & $\begin{array}{l}68(98.55) \\
1(1.45)\end{array}$ & $\begin{array}{l}31(100) \\
0(0)\end{array}$ & $\begin{array}{l}99(99) \\
1(1)\end{array}$ & 0.69 \\
\hline
\end{tabular}

Further, we determined the effect of blood transfusion and red blood concentrate on sternal infections and wound conditions. A trend was observed with the use of no blood products (blood transfusion, red blood concentrates) given postoperatively, the presence of sternal infections and wound dehiscence was not found in any patient. However, the use of blood transfusions and red bold concentrates didn't have statistical significance in the patients with or without sternal infections ( $\mathrm{p}=1$ and 0.08 respectively) and patients who had healthy and stable wounds or wound dehiscence $(\mathrm{p}=0.32$ and 0.29 respectively) (Table 6). We would like to add that in cases of coronary artery bypass grafting unilateral or bilateral internal mammary artery was harvested depending on the blockage of coronary arteries.

Table 6. The association of blood transfusion with the sternal wound complications.

\begin{tabular}{|c|c|c|c|c|c|c|c|c|c|}
\hline & & \multicolumn{2}{|c|}{ Blood transfusion } & \multirow{2}{*}{ Total } & \multirow{2}{*}{$\begin{array}{l}\mathbf{p} \\
\text { Value }\end{array}$} & \multicolumn{2}{|c|}{ Only red blood concentrate } & \multirow[t]{2}{*}{ Total } & \multirow{2}{*}{ P-value } \\
\hline & & No & Yes & & & No & Yes & & \\
\hline $\begin{array}{l}\text { Sternal infections } \\
\text { (superficial or deep) }\end{array}$ & $\begin{array}{l}\text { No } \\
\text { Yes }\end{array}$ & $\begin{array}{l}68(100) \\
0(0)\end{array}$ & $\begin{array}{l}30(93.75) \\
2(6.25)\end{array}$ & $\begin{array}{l}98(98) \\
2(2)\end{array}$ & 0.100 & $\begin{array}{l}71(100) \\
0(0)\end{array}$ & $\begin{array}{l}27(93.1) \\
2(6.9)\end{array}$ & $\begin{array}{l}98(98) \\
2(2)\end{array}$ & 0.08 \\
\hline $\begin{array}{l}\text { Sternum wound } \\
\text { condition }\end{array}$ & $\begin{array}{l}\text { Stable and healthy } \\
\text { wound } \\
\text { Wound dehiscence }\end{array}$ & $\begin{array}{l}68(100) \\
0(0)\end{array}$ & $\begin{array}{l}31(96.88) \\
1(3.13)\end{array}$ & $\begin{array}{l}99(99) \\
1(1)\end{array}$ & 0.32 & $\begin{array}{l}71(100) \\
0(0)\end{array}$ & $\begin{array}{l}28(96.55) \\
1(3.45)\end{array}$ & $\begin{array}{l}99(99) \\
1(1)\end{array}$ & 0.29 \\
\hline
\end{tabular}

The postoperative complications and infectious events such as pneumonia, sepsis, pneumothorax were absent. There was no death at the end of 30 days. 1 patient had a myocardial infarction, 1 patient had postoperative delirium, and 3 patients had impairment in renal functions. The mean duration of hospital stay and intensive care unit (ICU) stay was 8.53 (SD: 4.84) days and 3.58 (SD: 5.01) days respectively.

\section{Discussion}

The ideal sternal closure system should possess adequate mechanical strength, radiopacity, biocompatibility, removability when necessary, cost-effectiveness, no infection risks, and should provide stability to the sternum and apt fixation. [8] Currently, SS wire is the gold standard in median sternotomy, however it can cause severe morbidity due to its shortcomings. [9] In co-morbid conditions, patients face complications with the use of SS wire. For patients with soft and weaker bones or patients with osteoporosis, a wire can cut through the sternum with the pressure while coughing or performing any strenuous activity. Wire cutting through the sternum due to force, may require resurgery and further can be aggravated by infections like mediastinitis which can lead to mortality [1] Casha et al. showed that major sternal complications occur due to osteoporosis in $2 \%$ of the sternal closure procedures. [1]

ZipFix system which is studied here along with SS wire has superiority over SS wire in terms of the surface area of the sternum in contact with the ZipFix. As the ZipFix cable is wider [8], the available surface area between the sternum and ZipFix system is greater, which creates a lower pressure on the sternum and also has 5 times more resilience. [2] ZipFix is enveloped in the intercostal area of the sternum, bleeding can be reduced as compared to the standard technique in which the wire could cut through the sternum. [7] ZipFix system which is used in the combination with SS wire to fix the manubrium is used at 3 or 4 spaces. When mechanical 
tests of both SS wires and ZipFix systems are compared, SS wires' resistance to cut-through is much lesser than ZipFix's resistance to cut-through. Sternal wires are hand-tightened, so equivalent tension is not applied on each wire. So the chances of cut-through are increased. The sternal ZipFix system application instrument can allow steady tensioning along the length of the sternum. It has rounded edges that may reduce soft tissue irritation, and its PEEK material can be cut easily in case of an emergency, hence it can be easily removable. This material is MRI safe and isn't detected in Xrays. It can be used alone or in conjunction with traditional steel wire closures or other fixation systems. This technique has potential benefits such as flexibility, easy handling, capacity in closing the sternum with high stability, high biocompatibility, and low risk of glove puncture. [2]

ZipFix is compared to other materials for closure of the sternum system such as Sterna band, sternal plates, nylon bands, suture materials. It has been identified that the use of sternal plates is structurally better than SS wire and can reduce the risk of DSWI and mediastinitis. However, it failed due to greater operative times and expensive nature and can cause complications while emergent re-entry. A large cohort study by Schimmer et al. failed to gather the advantages of sternal plates. [10-13] There has been a hypothesis that greater surface area of broader suture materials can distribute the pressure on the sternum so that tension can be minimized with less risk of breaking the sternum and can provide adequate stability to the sternum. Attempts were made with polyethylene terephthalate ribbon, jacketed steel wires, and steel bands, but the difficulty in implantation and use and increased development of infection (such as mediastinitis) terminated their use. [9, 14] A few trials with Nylon bands showed an increased incidence of post-surgical complications. [15] Similarly, Sterna band can effectively fix and also decrease postoperative pain and length of hospital stay. [9, 16] The advantages of the ZipFix over the Sterna band are easy to use and lesser application time, and the reproducible tension of $200 \mathrm{~N}$ for each ZipFix. [2]

The predictive factors responsible for the development of DSWI are advanced age, obesity, diabetes, smoking, prolonged operative time, use of internal thoracic artery conduits, hemodialysis, reoperative pulmonary surgery, and prolonged cardiopulmonary bypass time. $[5,17,18]$ However, in this study, smoking history, age, and BMI of patients had no statistical significance over sternal dehiscence and sternal wound condition.

In a study by soroff et al, the mean age of the population undergoing median sternotomy was 65 years and the mean length of postoperative hospital stay was $13.9 \pm 3.4$ days. [9] Whereas Ozmen et al claimed that the mean length of postoperative stay for patients who developed sternal infections was 4-6 weeks [2] while this study suggests the mean length of the hospital stay was $8.53 \pm 4.84$ days. The combination of the ZipFix and steel wires has certainly reduced the length of the hospital stay postoperatively.

As seen in this study, the use of transfusions of blood products was associated with developing sternal infections or dehiscence. Melly et al had observed that the necessity of blood products (fresh frozen plasma, thrombocytes, and erythrocytes concentrate) was decreased postoperatively after the use of ZipFix for median sternotomy. [7] The Logistic Euro score should be lesser than $3 \%$ to have good survival rates. [2] This study claimed logistic euro score (the ZipFix bands and steel wire) $2.84 \pm 2.78 \%$, which is better than studies such as Melly et al, Graopow et al where only steel wire was used, where the logistic euro score (only steel wires) was found to be $12 \pm 15 \%$, [7] 15 $\pm 19 \%$ [2] respectively.

Various retrospective and prospective studies had tried to compare the efficacy and safety of ZipFix wires over SS wires. $\mathrm{Oh}$ et al. failed to observe any statistical significance in both groups (multifilament cable system and SS wires). Though there were a few cases of sternal bleeding in the cable tie sternum closure system, it was not statistically significant. [19] Melly et al demonstrated that though the incidence of DSWI or superficial wound infection diminished in the cable tie sternum closure group compared to the SS wire group, the reduction wasn't statistically significant. [7] However, Nezafati et al showed no statistically significant in overall sternal infections. Results were neutral for both groups (the ZipFix and SS wire). However, in a month follow-up, they found a significant reduction in sternal dehiscence in the ZipFix group $(\mathrm{P}<0.001)$. [6] Dunne et al. implicated that the sternal cable method helped in early extubation after cardiac surgeries, but didn't decrease DSWI. [20] Tam et al reported that ZipFix improved pain scores in patients and caused a statistically significant reduction in sternal dehiscence (ZipFix group (0) and wire group (5); $\mathrm{P}<.001$ ). Also, in another study, the use of the rigid plate fixation technique reduced the sternal complications and improved the sternal healing than wire cerclage. Tam et al suggested implementing the principle behind this study with ZipfFx to improve the outcomes [21, 22]

All these studies have claimed the reduction of postoperative complications but failed to demonstrate statistical significance. Several post-surgical complications (e.g., sternal dehiscence, sternal bleeding, infections, and prolong extubation time, among others) appear to be less in the sternal cable technique. The studies including the combination of the ZipFix and SS wires are used are unavailable in the literature. This study demonstrated that the use of the ZipFix system along with SS wire can reduce the shortcomings of SS wires as experienced by patients with the use of only SS wires.

\section{Limitation}

This is retrospective where a combination of wire sutures and cable ties were used; thereby, we couldn't comment on the efficacy and safety of the ZipFix as an independent method for sternal fixation.

\section{Conclusion}

In our study, the ZIPFIX system along with SS wires was convenient in terms of its application; there were no hardware failures leading to further complications. The postoperative outcomes in the sternal cable group were less 
frequent but not statistically significant with respect to age, BMI, use of blood products, and smoking behavior. The use of steel wire along with the ZipFix was effective and can be a substitute to simple SS wire. In order to demonstrate the superiority of the ZipFix over conventional methods, the ZipFix should be used alone and more randomized studies with more parameters and patients should be studied.

\section{References}

[1] Alhalawani. A review of sternal closure techniques. A review of sternal closure techniques. J Biomater Appl. 2013 Nov; 28: 483-97.

[2] Kubota H, Miyata H, Motomura N, Ono M, Takamoto S, Harii $\mathrm{K}$, et al. Deep sternal wound infection after cardiac surgery. J Cardiothorac Surg. 2013; 8: 132.

[3] Tang A. Novel application of vacuum assisted closure technique to the treatment of sternotomy wound infection. European Journal of Cardio-Thoracic Surgery. 2000; 17: 4824.

[4] Gårdlund B. Postoperative mediastinitis in cardiac surgery microbiology and pathogenesis. European Journal of CardioThoracic Surgery. 2002; 21: 825-30.

[5] Lu J. Risk factors for sternal wound infection and mid-term survival following coronary artery bypass surgery. European Journal of Cardio-Thoracic Surgery [Internet]. 2003; 23: $943-$ 9.

[6] Nezafati P, Shomali A, Kahrom M, Omidvar Tehrani S, Dianatkhah M, Nezafati MH. ZipFix Versus Conventional Sternal Closure: One-Year Follow-Up. Heart, Lung and Circulation. 2019; 28: 443-9.

[7] Melly L, Gahl B, Meinke R, Rueter F, Matt P, Reuthebuch O, et al. A new cable-tie-based sternal closure device: infectious considerations $\dagger$. Interactive CardioVascular and Thoracic Surgery. 2013; 17: 219-24.

[8] Stelly MM, Rodning CB, Stelly TC. Reduction in deep sternal wound infection with use of a peristernal cable-tie closure system: a retrospective case series. J Cardiothorac Surg. 2015; 10: 166 .

[9] Soroff HS, Hartman AR, Pak E, Sasvary DH, Pollak SB. Improved sternal closure using steel bands: early experience with three-year follow-up. Ann Thorac Surg. 1996 Apr; 61: $1172-6$.

[10] Sargent LA, Seyfer AE, Hollinger J, Hinson RM, Graeber GM. The healing sternum: A comparison of osseous healing with wire versus rigid fixation. The Annals of Thoracic Surgery. 199; 52: 490-4.
[11] Raman J, Lehmann S, Zehr K, De Guzman BJ, Aklog L, Garrett HE, et al. Sternal Closure With Rigid Plate Fixation Versus Wire Closure: A Randomized Controlled Multicenter Trial. The Annals of Thoracic Surgery. 2012; 94: 1854-61.

[12] Song DH, Lohman RF, Renucci JD, Jeevanandam V, Raman J. Primary sternal plating in high-risk patients prevents mediastinitis 2 . European Journal of Cardio-Thoracic Surgery. 2004; 26: 367-72.

[13] Schimmer C, Reents W, Berneder S, Eigel P, Sezer O, Scheld $\mathrm{H}$, et al. Prevention of Sternal Dehiscence and Infection in High-Risk Patients: A Prospective Randomized Multicenter Trial. The Annals of Thoracic Surgery. 2008; 86: 1897-904.

[14] McGregor WE, Payne M, Trumble DR, Farkas KM, Magovern JA. Improvement of sternal closure stability with reinforced steel wires. The Annals of Thoracic Surgery. 2003; 76: $1631-4$.

[15] LeVeen HL. Nylon-Band Chest Closure. Arch Surg. 1968; 96: 36.

[16] Bhattacharya S, Sau I, Mohan M, Hazari K, Basu R, Kaul A. Sternal Bands for Closure of Midline Sternotomy Leads to Better Wound Healing. Asian Cardiovasc Thorac Ann. 2007; 15: 59-63.

[17] Milano CA, Kesler K, Archibald N, Sexton DJ, Jones RH. Mediastinitis After Coronary Artery Bypass Graft Surgery: Risk Factors and Long-term Survival. Circulation. 1995; 92: 2245-51.

[18] Matros E, Aranki SF, Bayer LR, McGurk S, Neuwalder J, Orgill DP. Reduction in incidence of deep sternal wound infections: Random or real? The Journal of Thoracic and Cardiovascular Surgery. 2010; 139: 680-5.

[19] Oh YN, Ha KJ, Kim JB, Jung S-H, Choo SJ, Chung CH, et al. Multifilament Cable Wire versus Conventional Wire for Sternal Closure in Patients Undergoing Major Cardiac Surgery. Korean J Thorac Cardiovasc Surg. 2015; 48: 265-71.

[20] Dunne B, Murphy M, Skiba R, Wang X, Ho K, Larbalestier R, et al. Sternal cables are not superior to traditional sternal wiring for preventing deep sternal wound infection. Interact CardioVasc Thorac Surg. 2016; 22: 594-8.

[21] Tam D, Fremes S. Cable ties for chest closure: zipfix or zipfail? The Journal of Thoracic and Cardiovascular Surgery. 2018; 156: 1611.

[22] Allen K, Thourani V, Naka Y, Grubb K, Grehan J, Patel N, et al. Randomized, multicenter trial comparing sternotomy closure with rigid plate fixation to wire cerclage. The Journal of Thoracic and Cardiovascular Surgery. 2017; 153: 888-896. 